\title{
Therapeutic Options for Neonatal Acute Kidney Injury (AKI)
}

\author{
Ayesa N. Mian, MD ${ }^{1, *}$
}

David J. Askenazi, MD, MSPH'

Maroun J. Mhanna, MD, MPH

\author{
Address \\ *,1Department of Pediatrics, Division of Nephrology, University of Rochester \\ School of Medicine, 601 Elmwood Avenue, Box 777, Rochester, NY, 14642, USA \\ Email: ayesa_mian@urmc.rochester.edu \\ ${ }^{2}$ Department of Pediatrics, Division of Pediatric Nephrology, University of Ala- \\ bama at Birmingham, 1600 7th Avenue S, Lowder 516, Birmingham, AL, 35233, \\ USA \\ ${ }^{3}$ Pediatric Critical Care, Neonatal Perinatal Medicine, Case Western Reserve Uni- \\ versity, 2500 MetroHealth Drive, Cleveland, OH, 44109, USA
}

Published online: 20 April 2016

(C) Springer International Publishing AG 2016

This article is part of the Topical Collection on Pediatric Nephrology

Keywords Neonatal acute kidney injury (AKI) $\cdot$ Renal failure $\cdot$ CRRT $\cdot$ CVVH $\cdot$ Peritoneal dialysis $\cdot$ Renal support therapy · Fluid overload · Renal recovery • Dialysis - Theophylline · Nephrotoxic · Prevention · CARPEDIEM • NIDUS

\section{Opinion statement}

Acute kidney injury (AKI) occurs commonly in neonates and is associated with increased mortality. Recent advances in neonatal intensive care have resulted in improved patient survival, but this had not been paralleled by improved outcomes for neonates with AKI. Management of AKI remains supportive with no effective pharmacologic therapy to hasten the recovery process. Emphasis is placed on the prevention and minimization of further renal insults once AKI is established. Routine, regular assessments of renal function with identification of high risk patients and those with incipient AKI are essential so prevention strategies can be promptly initiated. Effective prevention strategies include prompt restoration/preservation of organ perfusion with fluid resuscitation to ensure an adequate effective circulating volume and inotropic support as needed to maintain hemodynamic stability while also avoiding/minimizing nephrotoxin exposure. In established AKI, efforts are focused on maintaining organ perfusion while closely monitoring cumulative fluid balance from admission and remaining cognizant of the need to minimize the degree of fluid overload as this has also been associated with poor outcomes. Continuous renal replacement therapy (CRRT) has become the preferred therapy for managing fluid overload and metabolic disturbances in critically ill children and adults but is still considered by many to be a heroic intervention for neonates due in large part to numerous technical challenges. The recent development of two miniaturized machines specifically designed to 
deliver CRRT and hemodialysis (HD) to neonates will hopefully change this paradigm making it easier for nephrologists and intensivists to safely offer and provide this therapy to neonates. Prospective studies are needed to assess whether early initiation of RRT with correction of fluid imbalance results in improved patient survival and renal recovery.

\section{Introduction}

\section{AKI epidemiology and definition}

Acute kidney injury (AKI) is now recognized to be a complex syndrome ranging in severity from mild reversible renal dysfunction to severe, oligoanuric failure. It is associated with increased mortality in the acute setting as well as an increased risk for CKD amongst survivors [1-3]. Using contemporary definitions, the incidence of AKI in neonates has been estimated to range from 16 to $72 \%$ based on the particular population studied (verylow-birth-weight infants, complex congenital heart disease, extracorporeal membrane oxygenation (ECMO), neonatal asphyxia) [4-8]. Conceptually, AKI signifies an abrupt decline in glomerular filtration accompanied by a loss of volume regulation and electrolyte/acid-base homeostasis as well as by an accumulation of nitrogenous waste products. Operationally, this has been much more challenging to define. Paradoxically, the incidence of AKI in critically ill patients has been increasing in the past few decades despite advances in critical care medicine and neonatology as these advances have resulted in improved patient survival but not in the development of effective treatments for AKI [9]. Care for patients with AKI remains supportive.

Research in the field of AKI has been hindered by lack of a consensus definition for AKI until relatively recently. In the past 15 years, three standardized working definitions for AKI (Risk, Injury, Failure, Loss, ESRD (RIFLE),/ pediatric RIFLE (pRIFLE), Acute Kidney Injury Network (AKIN), and Kidney Disease: Improving Global Outcomes (KDIGO)) have been proposed and validated to clinical outcomes in adults, but not in children or neonates $[1,9-11,12 \bullet]$. All are based on an absolute or relative increase in serum creatinine from an established baseline or by the development of oliguria. The pitfalls of a serum creatinine-based definition for AKI are well recognized and include the (a) influence of nonglomerular filtration rate (GFR) determinants (e.g., muscle mass, diet, hydration status); (b) lack of sensitivity to acute, small changes in renal function; and (c) excretion, albeit limited, by routes other than filtration (proximal tubular secretion and bacterial degradation in the gastrointestinal tract) $[13,14]$. Use of a creatininebased definition in neonates is further complicated by the unique physiology of neonates. Creatinine equilibrates across the placenta in utero which makes the serum creatinine at birth reflective of the mother's renal function not the infant's. Acute changes in volume status which happen over the first weeks of life affect the $S_{\mathrm{Cr}}$ measurement [15]. GFR at birth is low in newborns and increases during the first few weeks. In full-term infants, as the GFR increases, the serum creatinine gradually declines becoming reflective of the infant's renal function by 1 week of age as a new equilibrium is attained. In preterm infants, due to greater immaturity of the renal function, the serum creatinine will take weeks to reach its nadir $[16,17]$. Further, preterm infants often experience an initial rise in serum creatinine during the first few days of life before peaking and progressively declining $[18,19]$.

Recently, neonatologists and pediatric nephrologists have formed an international collaborative, the Neonatal Kidney Collaborative (NKC), to foster the conduction of well-designed studies which will advance our knowledge about the epidemiology, prevention, therapy, and outcomes of neonatal AKI. One of the first aims of the group is to develop and validate a uniform definition for neonatal AKI. The collaborative has proposed a working definition for AKI based on modifications to the KDIGO AKI definition Table 1 [5, 20••].

\section{Etiology and management overview}

Hypoperfusion is by far the leading cause of neonatal AKI occurring secondary to hypovolemia (e.g., placental abruption, excessive third spacing, insufficient fluid replacement), hypotension (e.g., vasodilation from sepsis), and hypoxemia (e.g., chronic lung disease, fluid overload, abruption). Prolonged hypoperfusion can result in parenchymal damage and acute tubular necrosis (ATN). Intrinsic renal injury can also result from infection (e.g., pyelonephritis), vascular insults (e.g., thrombosis), and exposure to nephrotoxins (e.g., NSAIDs/ aminoglycosides/contrast dye). Post-renal causes are much less common but crucial to recognize early as 


\section{Table 1. Neonatal AKI KDIGO classification}

\begin{tabular}{|c|c|c|}
\hline Stage & $\mathrm{SCr}$ & Urine output \\
\hline 0 & No change in $\mathrm{SCr}$ or rise $<0.3 \mathrm{mg} / \mathrm{Ll}$ & $\geq 0.5 \mathrm{mg} / \mathrm{kg} / \mathrm{h}$ \\
\hline 1 & $\begin{array}{l}\mathrm{SCr} \text { rise } \geq 0.3 \mathrm{mg} / \mathrm{dL} \text { within } 48 \mathrm{~h} \text { or } \mathrm{SCr} \text { rise } \geq 1.5-1.9 \times \text { reference } \mathrm{SCr}^{\mathrm{a}} \\
\text { within } 7 \text { days }\end{array}$ & $<0.5 \mathrm{ml} / \mathrm{kg} / \mathrm{h}$ for 6 to $12 \mathrm{~h}$ \\
\hline 2 & $\mathrm{SCr}$ rise $\geq 2.0-2.9 \times$ reference $\mathrm{SCr}^{\mathrm{a}}$ & $<0.5 \mathrm{ml} / \mathrm{kg} / \mathrm{h}$ for $\geq 12 \mathrm{~h}$ \\
\hline 3 & $\mathrm{SC}$ r rise $\geq 3 \times$ reference $\mathrm{SCr}^{\mathrm{a}}$ or $\mathrm{SCr} \geq 2.5 \mathrm{mg} / \mathrm{dL}^{\mathrm{b}}$ or receipt of dialysis & $\begin{array}{l}<0.3 \mathrm{ml} / \mathrm{kg} / \mathrm{h} \text { for } \geq 24 \mathrm{~h} \text { or anuria } \\
\quad \text { for } \geq 12 \mathrm{~h}\end{array}$ \\
\hline \multicolumn{3}{|c|}{ 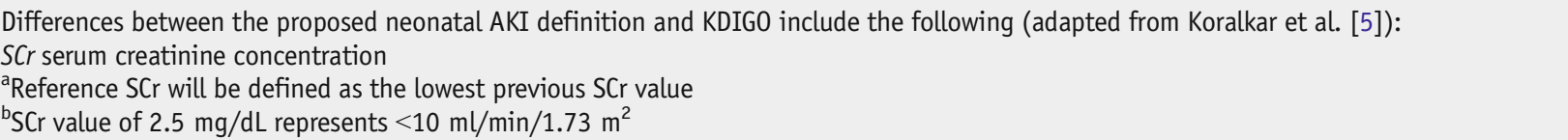 } \\
\hline
\end{tabular}

the cornerstone of therapy is relief of the obstruction. Post-renal causes of neonatal renal failure include congenital anomalies of the urinary tract (e.g., posterior urethral valves, ureteropelvic or ureterovesicle junction obstruction), neurogenic or medication-related urinary retention, and acquired obstructions such as fungal bezoars and nephrolithiasis.

Given the lack of effective therapy, and limited support options with current renal replacement therapies, emphasis is placed on (a) early recognition of high risk patients as well as those with incipient AKI and on (b) strategies to prevent of minimize progression of incipient AKI. Supportive care measures for developing or established AKI include the following:

(a) Treatment of the underlying process (e.g., antibiotics for sepsis, relief of obstruction);

(b) Fluid therapy to correct hypovolemia and vasopressors, as needed, to maintain adequate blood pressure and ensure tissue perfusion;

(c) Careful attention to fluid balance with use of fluid restriction, diuretics, and/or renal replacement therapy to minimize/manage fluid overload;

(d) Electrolyte supplementation and/or binders to restore electrolyte and acid-base homeostasis;

(e) Provision of adequate nutrition to promote healing;

(f) Avoidance of further renal insults including exposure to nephrotoxins (medications/contrast dye), development of intravascular volume depletion especially during recovery phase of acute tubular necrosis (ATN), as well as the development of significant cumulative fluid overload;

(g) Dose adjustment for medications which are nephrotoxic and for those which are eliminated by the kidney;

(h) Renal replacement therapy to prevent further fluid accumulation, restore euvolemia and metabolic balance, as well as to allow provision of adequate nutrition/blood products/ medications.

\section{Medical management of AKI}

\section{Fluid and vasopressor therapy}

Preventive strategies for AKI begin with early identification of high risk patients coupled with careful attention to correction of hypovolemia, if present, and maintenance of hemodynamic stability. Early assessment for cardiogenic shock in the neonate is critical and necessitates the initiation of a prostaglandin $\mathrm{E}_{1}\left(\mathrm{PGE}_{1}\right)$ infusion to maintain ductal patency pending evaluation for complex duct-dependent congenital heart disease. In contrast, neonates with hypovolemic or septic shock require rapid fluid resuscitation to re-establish and/or preserve organ perfusion and function. Fluid and medications to increase blood pressure need to be aggressively but judiciously administered to achieve specific physiologic endpoints (mean arterial pressure, CVP, oxygen saturation, urine output) while minimizing significant cumulative fluid overload. Implementation of protocoldriven early-goal-directed therapy has been associated 
with improved patient survival as well as a lower incidence of AKI [21, 22].

Aside from the scenario of acute blood loss with hemorrhagic shock, KDIGO AKI guidelines recommend isotonic crystalloid solutions over colloid solutions for rapid volume expansion. Use of balanced crystalloid solutions simulating plasma (e.g., Lactated Ringer's, PlasmaLyte) may be preferable to unbalanced crystalloid solutions (NS) for volume resuscitation in adults with sepsis as the hyperchloremic metabolic acidosis resulting from aggressive resuscitation with NS has been associated with greater renal dysfunction; however, it is unclear whether use of buffered solutions results in improved outcomes $[23,24]$. Insufficient data exist to make any recommendation regarding choice of crystalloid solution for volume resuscitation in children and neonates.

Vasoactive support is indicated if hypotension persists despite correction of hypovolemia and initiation of $\mathrm{PGE}_{1}$ infusion in the case of duct-dependent congenital heart disease with cardiogenic shock. The KDIGO AKI guidelines emphasize that vasoactive support should not be withheld in volume-resuscitated patients due to concerns that vasoconstriction will reduce renal perfusion. In the volume replete patient, mean arterial pressure and perfusion increase with inotropic support [25••] Insufficient data exist to suggest superiority of one vasopressor over another in the prevention/ management of AKI. Dopamine and dobutamine are commonly used in neonates. At low doses $(5 \mathrm{mcg} / \mathrm{kg})$, dopamine binds to dopaminergic receptors in the kidney resulting in vasodilatation and improved blood flow. In the past, low-dose dopamine was frequently used with the belief that it offered some renoprotection. However, several randomized, double-blind, placebo controlled trials in adults/children have failed to show any renal benefit from dopamine and some studies have shown it may be harmful. The use of low-dose dopamine to prevent or treat AKI is therefore not recommended in children/adults $[25 \bullet \bullet, 26]$. At higher doses, dopamine has inotropic and alpha adrenergic effects resulting in increased cardiac output and vasoconstriction. Epinephrine is used for fluid refractory-dopamineresistant shock. The 2007 Updated Clinical Practice Parameters for hemodynamic support of neonatal shock provides details regarding pressor/inotrope support [21].

Despite aggressive efforts to improve hemodynamic status, prerenal AKI may still progress to intrinsic AKI/ acute tubular necrosis. Accurate serial volume assessments are essential for fluid management in AKI. In a recent review, Goldstein outlined the three phases of fluid management in AKI: resuscitation, maintenance, and recovery $[27 \bullet \bullet]$. The goal in phase 1 , fluid resuscitation, is the prompt administration of fluid to restore effective circulating volume and re-establish/maintain organ perfusion as well as function. Phase 1 may overlap with phase 2 , the maintenance phase, as the hypovolemia due to capillary leak from sepsis may persist for several days. In phase 2, the focus shifts to the maintenance of fluid balance allowing for the provision of needed therapies (medications/blood products/and adequate nutrition) while simultaneously minimizing cumulative fluid overload. This requires careful attention to daily weights, total input (including flushes), and total output (insensible water losses, urine output, gastrointestinal losses, other losses such as chest tube, etc). Daily assessments of fluid balance as well as cumulative fluid balance from admission should be monitored. Cumulative fluid overload exceeding $10 \%$ has been associated with significantly higher mortality in adults and children [28-31]. In the oliguric patient, strategies to maintain fluid balance include fluid restriction, diuretic therapy, and renal replacement therapy. In neonates, fluid restriction is often implemented when diuretics fail as renal replacement therapy is much more challenging to provide to this group of patients and still often considered heroic.

However, advances in the past decade have made renal replacement therapy (RRT) much more feasible for neonates. (See "Renal support therapies" section) In phase 3, the recovery phase, attention is directed towards fluid removal balancing the competing goals of rapid diuresis to optimize pulmonary status and weaning off the ventilator with the goal of preventing further renal ischemic insults secondary to overaggressive diuresis. Early initiation of RRT with the use of inotropes to maintain hemodynamic stability has been hypothesized to improve outcomes [30].

Finally, it is also important to remember that AKI in neonates is often non-oliguric, which often hides its presence to the clinician. High-output renal failure is also often seen during the polyuric phase of ATN, following relief of a urinary tract obstruction (post-obstructive diuresis), and in those with nephrotoxin injury (e.g., aminoglycosides). Meticulous attention to fluid balance is again essential with recognition that protocolized fluid administration based on gestational age and weight often requires readjustment. Development of intravascular volume depletion at this time can hinder 
renal recovery and potentially result in another episode of AKI.

\section{Theophylline}

AKI is a common complication of infants experiencing severe perinatal asphyxia with incidence rates of over $50 \%$ reported in several studies [32-34]. During acute hypoxemia, adenosine levels increase as ATP hydrolysis exceeds synthesis resulting in intrarenal vasoconstriction and a fall in GFR. Four randomized controlled trials of severely asphyxiated neonates, three involving term and one involving preterm infants, found that a single prophylactic dose of theophylline, an adenosine receptor antagonist, administered within $1 \mathrm{~h}$ of birth had a renoprotective effect $[35-38,39 \bullet]$. Theophylline treatment was associated with a significantly higher GFR and urine output as well as less cumulative fluid overload. KDIGO AKI guidelines for the management of AKI suggest that a single dose of theophylline can be considered in severely asphyxiated infants at high risk for AKI [25••]. Potential long-term renal and neurodevelopmental sequelae associated with the use of theophylline in this setting, however, have not been well studied. Additionally, the effect of prophylactic theophylline in the setting of therapeutic hypothermia, now the standard treatment for severely asphyxiated infants, has not been studied. Therefore, prophylactic theophylline should be used cautiously.

Therapeutic hypothermia is also thought to potentially offer some renoprotection by reducing metabolic demand and oxygen consumption. In adults experiencing out-of-hospital cardiac arrests, use of therapeutic hypothermia has been associated with decreased neurologic morbidity and mortality [40]. However, the impact of hypothermia on renal function in severely asphyxiated neonates has not been prospectively studied. In a recent retrospective review of severely asphyxiated newborns treated with therapeutic hypothermia, Selewski et al. reported an AKI incidence of $38 \%$, somewhat lower than that reported in historical studies performed before therapeutic hypothermia became the standard care [8].

\section{Medication management}

\section{Nephrotoxic medication exposure}

Avoidance/minimization of nephrotoxin exposure is another cornerstone in the prevention of AKI. Rhone et al. reported an overall high rate of nephrotoxic medication exposure in very-low-birth-weight (VLBW) infants with $87 \%$ receiving one or more nephrotoxic medications and $26 \%$ developing AKI. Further, those with AKI were treated with more nephrotoxic medications per day than those without AKI [41•]. The most common exposures included aminoglycosides (86\%), indomethacin (50\%), and vancomycin (29\%). Risk factors for aminoglycoside nephrotoxicity included concurrent use of other nephrotoxic medications, high drug levels, prolonged treatment courses, and repeated treatment courses within a short time period. Intravascular volume depletion and pre-existing renal dysfunction may be additional risk factors. KDIGO guidelines for AKI management now recommend aminoglycosides not to be used unless appropriate, less nephrotoxic alternatives do

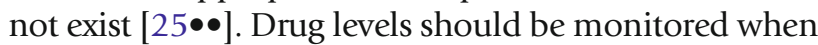
aminoglycoside therapy is necessary for more than $24 \mathrm{~h}$ with multiple daily dosing and $48 \mathrm{~h}$ with single daily dosing.

Indomethacin is another common exposure for preterm infants. Exposure may begin prenatally when used for tocolysis or postnatally when used for intraventricular hemorrhage prophylaxis or treatment of symptomatic patent ductus arteriosus (PDA). Management of the hemodynamically significant PDA poses a clinical conundrum as AKI can result from treatment for the PDA as well as from the PDA itself as blood is shunted away from the kidneys. Following fluid restriction, first line treatment for a persistent hemodynamically significant PDA includes indomethacin or ibuprofen, the latter reported to be less nephrotoxic [42, $43]$. Both are contraindicated in moderate to severe AKI but can be administered when mild AKI exists. Although the treatment can be nephrotoxic, it can paradoxically improve renal perfusion and function by promoting ductal closure. Studies that explore the use of these medications for PDA closure should include short- and long-term kidney outcomes in these infants.

Recently, there has been increased interest in the use of acetaminophen as an alternative therapy for medical closure of PDA, particularly in neonates for whom NSAIDs are contraindicated. Acetaminophen is hypothesized to constrict the ductus by inhibiting the peroxidase portion of the prostaglandin synthase enzyme. Although a recent metaanalysis suggests acetaminophen is as effective as ibuprofen for ductal closure, the majority of included studies were uncontrolled and not of high 
quality [44]. Randomized controlled trials as well as additional safety and efficacy data are needed before this therapy can be recommended.

\section{Medication dosing}

Medication dosing in critically ill neonates with AKI can be challenging. Nephrotoxic medications as well as those eliminated by the kidney may require dose reductions. On the other hand, hydrophilic medications may need to be dose adjusted upwards if there is significant fluid overload or decreased protein binding as both of these can increase the volume of distribution. Drugs with low protein binding and low volume of distribution will get cleared with dialysis. Monitoring drug levels when available can help guide dosing as serum creatinine does not accurately reflect renal function when not in a steady state [45].

\section{Diuretics}

Loop diuretics are frequently administered in the setting of oliguric AKI in hopes of converting to non-oliguric AKI which may, in turn, be misconstrued as an improvement in renal function. The increase in urine output resulting from administration of loop diuretics results from inhibition of sodium and water transport in the ascending limb of the loop of Henle and not from improvement in glomerular filtration. Loop diuretics continue to be administered commonly in clinical practice and may make fluid and electrolyte management easier thereby reducing the degree of fluid retention. However, no clinical studies unequivocally demonstrate an improvement in clinical outcomes (e.g., higher patient or renal survival, time to renal replacement therapy) to support the ongoing use of loop diuretics in this setting [46]. Indeed, there is some data to suggest it may even be harmful [47].

In neonates with AKI, the volume requirements for even maximally concentrated meds, blood products, and adequate nutrition often surpass what the infant is able to excrete making fluid restriction impractical in many situations. Diuretics are commonly prescribed in neonatal AKI despite the lack of evidence showing improved outcomes. The technical challenges for providing renal replacement therapy to neonates, especially premature, extremely low birth-weight (ELBW) infants, along with physician bias and experience, often make renal replacement therapy a "last resort" consideration. However, newer technology designed to specifically support neonates with minimal complications will soon be available to assist with fluid management in the NICU.
Furosemide is used most frequently and can be administered in a bolus or continuous fashion. It is highly protein bound and actively secreted by organic acid transporters in the proximal tubule. Once it gains entrance to the urinary space, it acts on the Na-K-Cl cotransporter located on the luminal surface of cells in the ascending limb of the loop of Henle where it inhibits sodium and water reabsorption. Decreased binding of loop diuretics to albumin, as can occur with hypoalbuminemia, hyperbilirubinemia, and/or coadministration of other medications which are highly protein bound, can decrease the effectiveness of the loop diuretics by impeding their delivery to the urinary space. Bumetanide is considered more potent than furosemide and enters the urinary space by proximal tubular secretion as well as by diffusion across tubular cell membranes because it is lipid soluble. In a recent study of oliguric AKI in preterm infants, Oliveros et al. found bumetanide more effective than furosemide for increasing urine output [48], but the results may have been biased by use of non-equivalent doses of furosemide and bumetanide [49]. Merheb et al. similarly found that bumetanide effectively increased urine output in ELBW infants, but this was associated with an increase in serum creatinine [50]. Although the use of diuretics cannot be justified in terms of improved patient or renal survival, they can be helpful for fluid management if oliguric AKI can be converted to nonoliguric AKI. This must be done cautiously however as aggressive use of diuretics can result in intravascular volume depletion with subsequent re-injury to the kidney.

\section{Renal support therapies}

The optimal modality, dosing, and timing for initiation of renal replacement therapy are unknown. The choice of modality-peritoneal dialysis (PD), hemodialysis (HD), or continuous renal replacement therapy (CRRT) - is often based on physician experience and preference as well as institutional resources.

\section{Peritoneal dialysis}

PD has generally been the preferred form of RRT for neonates as it is technically easier to establish and maintain dialysis access and it does not require anticoagulation, specialized machinery, or specialized nursing. The gradual ultrafiltration and metabolic clearance make it well tolerated but less suited for urgent situations such as hyperkalemia or pulmonary edema. PD efficiency can also be limited by the patient's 
peritoneal membrane transport characteristics as well as the proportionally larger tubing dead space relative to the fill volume in neonates. The immature skin and paucity of subcutaneous tissue place the preterm infant at increased risk for exit site leakage which, if it occurs, not only renders PD futile but also increases the risk for infection. Finally, PD is contraindicated in the presence of conditions which compromise the integrity of the abdominal wall such as omphalocele or recent abdominal surgery.

Performing PD in infants can be laborious as the small fill volume required often precludes the use of automated cyclers necessitating the use of closed-circuit manual setups (e.g., Gesco Dialy-Nate ${ }^{\circledR}$ ). The Baxter Home Choice ${ }^{\circledR}$ cycler is reported to deliver fill volumes as small as $60 \mathrm{ml}$, but in practice, alarms are frequently experienced with fill volumes less than $100 \mathrm{ml}$.

Alparslan et al. in 2012 described their experience providing PD to preterm and term neonates (GA 27-41 weeks; weight 1000-3800 g) using an Baxter Home Choice ${ }^{\circledR}$ cycler adapted for very low fill volumes and using a single cuffed Tenckhoff neonatal straight catheter placed percutaneously with one or two sutures closing the skin [51]. The Baxter Home Choice ${ }^{\circledR}$ cycler was adapted with an infusion pump to allow delivery of fill volumes less than $60 \mathrm{ml}$, the smallest fill volume which the cycler can deliver. Similar to other published reports, they experienced high complication ( $26 \%$ ) and mortality rates ( $59 \%)$.

As improvements in neonatal intensive care have resulted in improved survival of ELBW infants, the limits of RRT are continually challenged. The overall incidence of AKI in preterm infants is high and appears to be inversely correlated to gestational age and birth weight [5]. In recent years, pediatric nephrologists, neonatologists, and pediatric surgeons have developed innovative strategies using available equipment and technology to manage AKI in these vulnerable patients. Commercially available PD catheters are generally too large for ELBW infants leading practitioners to improvise dialysis access with the use of non-traditional catheters such as IV cannulas, silicone drains, and vascular catheters [5254]. Angiocaths are attractive because they do not require a stylet for placement thereby reducing the risk of visceral injury. However, the flexibility of angiocaths makes them more prone to kinking with subsequent catheter malfunction.

Harshman et al. [55] described their successful experience providing cycling PD to an ELBW infant (BW $830 \mathrm{~g}$ ) for 3 weeks using a standard $8 \mathrm{~cm}$ temporary
PD catheter placed in a non-traditional location-the left upper quadrant. The higher insertion allowed effective placement given the catheter length relative to the infant's torso length. To prevent leakage, the catheter was secured with a purse-string suture at the posterior rectus sheath with running sutures used to close the subcutaneous tissue and skin.

Assuming access can be achieved, the efficiency of continuous cycling PD (CCPD) is still low compared to HD or CRRT. Use of low initial fill volumes helps reduce the chances for leakage but also limits the clearance and ultrafiltration achieved. To improve the efficiency of PD, Raaijmakers et al. performed continuous flow peritoneal dialysis (CFPD) in neonates and children with AKI [56]. First introduced in the 1950s for adults, CFPD theoretically can improve the efficiency of PD by providing a continuous flow of dialysate through the peritoneal cavity. The continuous flow of dialysate provides a high concentration and osmotic gradient between the plasma and dialysate thereby maximizing solute mass transfer and ultrafiltration. Elimination of the catheter dead space associated with cyclical PD also theoretically helps improve the efficiency of PD for patients requiring small fill volumes. CFPD can be performed using two peritoneal catheters, one for continuous inflow of fresh dialysate and the other for drainage of used dialysate. Careful attention is required when placing the two catheters to allow sufficient distance between them so as to minimize recirculation. In a small study of six infants/ toddlers ranging in age from 11 days to 2.5 years, the authors report significantly higher clearance and ultrafiltration with CFPD compared to conventional cycling PD. Mean urea clearance $15 \mathrm{ml} / \mathrm{min} / 1.73 \mathrm{~m}^{2}$ vs $5 \mathrm{ml} /$ $\min / 1.73 \mathrm{~m}^{2}$; mean ultrafiltration (UF) $1.8 \mathrm{ml} / 1.73 \mathrm{~m}^{2}$ vs $0.2 \mathrm{ml} / 1.73 \mathrm{~m}^{2}$. The authors report no major complications but did experience some issues with inflow and nutrient loss [56].

\section{Hemodialysis and CRRT}

Intermittent HD and CRRT are more efficient than peritoneal dialysis and allow for more predictable fluid removal.

CRRT is preferred over HD for critically ill, hemodynamically unstable patients as it allows gradual, adjustable fluid, solute, and waste product removal.

However, use of both are limited in neonates due to the challenges of establishing vascular access as well as the technical and safety limitations of currently available machines. Achieving and maintaining a wellfunctioning vascular access is paramount for both HD 
and CRRT. Small double lumen catheters ( $5 \mathrm{Fr}$ ) are available but may not adequately support CRRT. In the prospective pediatric CRRT (ppCRRT) registry, when CRRT was provided using 5-Fr catheters, circuit lifespan was less than $20 \mathrm{~h}$ even with anticoagulation [55]. Seven Fr double lumen catheters, particularly when placed in the internal jugular vein, have been used successfully $[57 \bullet, 58]$. Ultrasound can be used to assess blood vessel caliber and aid in the selection of an appropriate-sized catheter. Placement of an overly large diameter catheter can be associated with vascular occlusion, thrombosis, and stenosis, which may have long-term implications for future dialysis access. El Masri et al. describe their experience successfully providing CRRT in neonates with cardiac disease and limited vascular options by use of two single lumen catheters (hemostasis valve sheaths) placed in two separate central veins. The valve sheaths provided adequate blood flow for CRRT, but the thin walls of the sheath were prone to kinking with subsequent introduction of air into the circuit. The authors propose the use of two 5-Fr single lumen continuous arteriovenous hemofiltration (CAVH) catheters which have more sturdy walls [59].

Currently available HD and CRRT machines were designed for adults and modified for use with children but are not currently FDA approved for children under $20 \mathrm{~kg}$. Machines can be set up with smaller circuits/filters, and roller pumps can be calibrated to provide blood flow rates as low as $30 \mathrm{ml} / \mathrm{min}$. Ideally, the circuit volume should be less than $10 \%$ of the patient's effective circulating volume to minimize hemodilution and hemodynamic instability. Even the smallest circuits currently available exceed this parameter for neonates necessitating the use of a blood prime which, in turn, is associated with the risks of infection, transfusion reaction, potassium and citrate load, sensitization due to HLA exposure, thrombocytopenia, hypocoagulabiltiy state, and the bradykinin release syndrome if an AN-69 hemofilter is used [60].

Finally, although HD and CRRT allow for programmable and more precise ultrafiltration control in adults and older children compared to PD, this does not hold true for neonates due to the relative imprecision of the scales for small volumes typical for neonates. For example, ultrafiltration accuracy may differ by $\pm 30 \mathrm{ml} / \mathrm{h}$ from the actual fluid removal with current machines. This imprecision represents $\sim 1 \%$ of the blood volume for a $70-\mathrm{kg}$ adult in comparison to $~ 12 \%$ for a $3-\mathrm{kg}$ neonate.

Clearly, to safely provide HD and CRRT to neonates, there is a need for specialized equipment designed with low circuit volumes which run at slow blood flow rates and which are associated with more precise UF scales. A small circuit ultrafiltration machine, Aquadex ${ }^{\circledR}$, has been adapted to provide continuous venovenous hemofiltration $(\mathrm{CVVH})$ in neonates. Collaboration between experts in the field of pediatric nephrology and industry within the past decade has also led to the exciting development of two miniaturized machines-Newcastle Infant Dialysis and Ultrafiltration System (NIDUS ${ }^{\circledR}$ ) and Cardio-Renal Pediatric Dialysis Emergency Machine (CARPEDIEM ${ }^{\circledR}$ )—capable of providing renal replacement therapy to neonates.

Askenazi et al. recently published a case series of CRRT on infants and small children performed by adapting the Aquadex ${ }^{\circledR}$ machine for CRRT. With this machine which has an extracorporeal volume of $33 \mathrm{ml}$, cardiovascular instability during CRRT initiation was minimal, even in severely critically ill patients $[61 \bullet \bullet]$.

NIDUS ${ }^{\circledR}$ provides hemodialysis to infants as small as $800 \mathrm{~g}$ (range $800 \mathrm{~g}-8 \mathrm{~kg}$ ). Unlike conventional HD machines which are driven by roller pumps, the NIDUS ${ }^{\circledR}$ is a syringe-driven machine which withdraws a small amount of blood (5-12.5 ml), passes it through a dialysis filter, and then returns it to the patient. The circuit volume has been reduced to less than $10 \mathrm{ml}$ obviating the need for a blood prime. UF rates in the range of $0-60 \mathrm{ml} / \mathrm{h}$ can be achieved with microliter precision of UF control. Use of this innovative technology allows treatment to be performed using a single lumen central venous line for up to $24 \mathrm{~h}$ at a time. Conventional HD may not be well tolerated by neonates due to rapid fluid and electrolyte shifts within a short period. However, the NIDUS ${ }^{\circledR}$ machine allows treatment to continue for up to $24 \mathrm{~h}$ making metabolic clearance and ultrafiltration more gradual and potentially better tolerated. It has been successfully

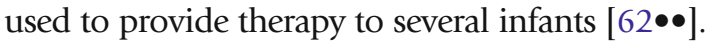

CARPEDIEM $^{\circledR}$ is a pump-driven machine which was designed to provide multiple therapies, including CRRT, to infants $2.5-10 \mathrm{~kg}$ in size. Though it requires a double lumen catheter, it was developed for use with small catheters (4-4.5 Fr). The extracorporeal circuit volume has been reduced to $27 \mathrm{ml}$ excluding the filter which may range in volume from 27.2 to $41.5 \mathrm{ml}$ (three filters available with surface areas of $0.075,0.15$, and $0.25 \mathrm{~m}^{2}$ ). Blood flow rates ranging from 5 to $50 \mathrm{ml} / \mathrm{min}$ can be achieved with excellent accuracy (error $<10 \%$ ) using miniaturized roller pumps which reportedly cause little microhemolysis. Gravimetric control along with improved UF scale sensitivity to $1 \mathrm{~g}$ provides more accurate fluid balance. CARPEDIEM ${ }^{\circledR}$ was licensed in Italy in 2013 and as of February 2016, 45 infants have been 
supported with CARPEDIEM ${ }^{\circledR}$ (verbal communication with Claudio Ronco). CARPEDIEM ${ }^{\circledR}$ has the additional advantage of being able to provide multiple extracorporeal therapies including continuous venovenous hemofiltration/continuous venovenous hemodialysis/ continuous venovenous hemodiafiltration (CVVH/ CVVHD/CVVHDF), plasma exchange, blood exchange, single pass albumin dialysis $[63,64 \bullet \bullet, 65]$.

\section{ECMO and CRRT}

Critically ill neonates requiring ECMO often have multiorgan system failure including renal dysfunction. The incidence of AKI is high in this population, exceeding $50 \%$ in several series $[7,66 \bullet, 67]$. The systemic inflammatory response resulting from exposure of the blood to the extracorporeal circuit and the ensuing capillary leak contribute both to the development of AKI and fluid overload. Increasing cumulative fluid overload leads to visceral edema [68] and has been shown in several studies to be associated with a longer ECMO course as well as increased mortality [69, 70]. Improvements in fluid balance and reversal of fluid overload is associated with improved survival, better lung function, and shorter time to decannulation [70-72]. A more recent study by Selewski et al. confirms the association between the degree of fluid overload and mortality in children on ECMO but did not find that reducing the degree of fluid overload once established resulted in improved survival suggesting the timing and duration of fluid accumulation are critical [73•]. ELSO guidelines now recommend minimizing the degree of fluid overload and trying to maintain euvolemia. (Extracorporeal Life Support
Organization Guidelines. Available at http:///www. elso.med.umich.edu/Guidelines.html). Indeed, in a survey of ELSO centers, Askenazi et al. found that the most common indication for initiating RRT while on ECMO is fluid overload ( $43 \%$ of centers) with another $16 \%$ of

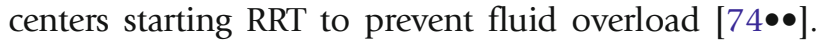
CRRT is used most frequently in conjunction with ECMO and can easily be performed by incorporation of either an in-line hemofilter or CRRT machine into the ECMO circuit. Refer to the review by Askenazi et al. for details regarding technical considerations (e.g., location of filter/machine placement) when combining these therapies $[74 \bullet \bullet]$.

Aggressive fluid removal with CRRT during ECMO can be limited by the development of hypotension and/ or excessive negative pressure in the venous limb of the ECMO circuit. Sasser et al. recently published their favorable experience adding PD to enhance fluid removal in post op cardiac neonates on ECMO who were already receiving CVVHF/CVVHD. The additional fluid removal by PD is thought to potentially be better tolerated because it is not directly lost from the macrocirculation but rather from the capillaries within the peritoneal membrane [75].

Diuretics, often employed during ECMO to minimize fluid overload, must be administered prudently as their use can increase the risk for prerenal AKI and ATN by masking oliguria secondary to intravascular volume depletion. In a recent study, loop diuretics were found to be an independent risk factor for AKI in children on ECMO receiving pre-emptive continuous hemofiltration [76].

\section{Conclusion}

Neonates are especially vulnerable to the development of AKI which, in turn, is associated with a high mortality. Despite advances in intensive care medicine, promising treatments to ameliorate AKI have not materialized. Although no specific pharmaceutical interventions are FDA approved for the treatment of AKI, the clinician can make important decisions that can ultimately impact outcomes. First, identification of patients at risk should be made by increasing provider awareness about AKI together with regular monitoring of renal function. Second, strategies to prevent poor outcomes in those at risk should be considered, including screening for sepsis, avoiding nephrotoxic medications, and optimizing hemodynamics. Close attention to hemodynamic status is critical and necessitates prompt, aggressive administration of fluids and pressors to restore/maintain visceral perfusion while simultaneously minimizing 
cumulative fluid overload. If the patient cannot tolerate the fluid necessary for nutrition and medications, CRRT should be considered.

CRRT is increasingly being used to manage fluid overload and metabolic disturbances in critically ill children and adults with studies now focusing on outcomes based on the timing of CRRT initiation. Provision of CRRT to neonates, however, continues to lag due in large part to numerous technical challenges associated with use of existing machinery which was designed to provide therapy to adults. The dedication of our European colleagues addressing these technical concerns is laudable and has resulted in two miniaturized machines which have been successfully used to safely provide therapy to several neonates. However, these machines are not yet universally available and in the USA, practitioners continue to adapt existing machinery in an effort to provide this therapy to neonates. The community has a moral obligation to assure that safe and effective therapies are available for the smallest infants, and collaboration from regulatory bodies such as the FDA, industry, and academics (both pediatric nephrologists and neonatologists) will be vital. Prospective studies will be needed to determine which babies, at which time points, are most likely to benefit from the therapies. Such efforts will likely improve patient survival and renal recovery.

\section{Compliance with Ethical Standards}

\section{Conflict of Interest}

Ayesa N. Mian and Maroun J. Mhanna declare that they have no conflict of interest.

David J. Askenazi has received personal fees from Baxter, BTW, and the Acute Kidney Injury Foundation outside of the submitted work.

Human and Animal Rights and Informed Consent

This article does not contain any studies with human or animal subjects performed by any of the authors.

\section{References and Recommended Reading}

Papers of particular interest, published recently, have been highlighted as:

- Of importance

$\bullet \quad$ Of major importance

1. Akcan-Arikan A, Zappitelli M, Loftis LL, Washburn KK, Jefferson LS, Goldstein SL. Modified RIFLE criteria in critically ill children with acute kidney injury. Kidney Int. 2007;71(10):1028-35.

2. Askenazi DJ, Feig DI, Graham NM, Hui-Stickle S, Goldstein SL. 3-5 year longitudinal follow-up of pediatric patients after acute renal failure. Kidney Int. 2006;69(1):184-9.

3. Mammen C, Al Abbas A, Skippen P, Nadel H, Levine D, Collet JP, et al. Long-term risk of CKD in children surviving episodes of acute kidney injury in the

intensive care unit: a prospective cohort study. Am J Kidney Dis. 2012;59(4):523-30.

4. Askenazi DJ, Koralkar R, Hundley HE, Montesanti A, Patil N, Ambalavanan N. Fluid overload and mortality are associated with acute kidney injury in sick near-term/term neonate. Pediatr Nephrol. 2013;28(4):661-6.

5. Koralkar R, Ambalavanan N, Levitan EB, McGwin G, Goldstein S, Askenazi D. Acute kidney injury reduces survival in very low birth weight infants. Pediatr Res. 2011;69(4):354-8. 
6. Alabbas A, Campbell A, Skippen P, Human D, Matsell D, Mammen C. Epidemiology of cardiac surgeryassociated acute kidney injury in neonates: a retrospective study. Pediatr Nephrol. 2013;28(7):1127-34.

7. Gadepalli SK, Selewski DT, Drongowski RA, Mychaliska GB. Acute kidney injury in congenital diaphragmatic hernia requiring extracorporeal life support: an insidious problem. J Pediatr Surg. 2011;46(4):630-5.

8. Selewski DT, Jordan BK, Askenazi DJ, Dechert RE, Sarkar S. Acute kidney injury in asphyxiated newborns treated with therapeutic hypothermia. J Pediatr. 2013;162(4):725-729.e1.

9. Selewski DT, Cornell TT, Heung M, Troost JP, Ehrmann $\mathrm{BJ}$, Lombel RM, et al. Validation of the KDIGO acute kidney injury criteria in a pediatric critical care population. Intensive Care Med. 2014;40(10):1481-8.

10. Bellomo R, Ronco C, Kellum JA, Mehta RL, Palevsky P, Acute Dialysis Quality Initiative workgroup. Acute renal failure - definition, outcome measures, animal models, fluid therapy and information technology needs: the Second International Consensus Conference of the Acute Dialysis Quality Initiative (ADQI) Group. Crit Care. 2004;8(4):R204-12.

11. Mehta RL, Kellum JA, Shah SV, Molitoris BA, Ronco C, Warnock DG, et al. Acute Kidney Injury Network: report of an initiative to improve outcomes in acute kidney injury. Crit Care. 2007;11(2):R31.

12. Kellum JA, Lameire N, KDIGO AKI Guideline Work Group. Diagnosis, evaluation, and management of acute kidney injury: a KDIGO summary (part 1). Crit Care. 2013;17(1):204.

Review summarizing key aspects of the 2012 KDIGO AKI Guidelines including AKI definition and staging, evaluation, and non-dialytic management.

13. Mitch WE, Walser M. A proposed mechanism for reduced creatinine excretion in severe chronic renal failure. Nephron. 1978;21(5):248-54.

14. Dunn SR, Gabuzda GM, Superdock KR, Kolecki RS, Schaedler RW, Simenhoff ML. Induction of creatininase activity in chronic renal failure: timing of creatinine degradation and effect of antibiotics. Am J Kidney Dis. 1997;29(1):72-7.

15. Askenazi D, Saeidi B, Koralkar R, Ambalavanan N, Griffin R. Acute changes in fluid status affect the incidence, associative clinical outcomes and urine biomarker performance in premature infants with acute kidney injury. Pediatric nephrology (Berlin, Germany) in press.

16. Schwartz GJ, Feld LG, Langford DJ. A simple estimate of glomerular filtration rate in full-term infants during the first year of life. J Pediatr. 1984;104(6):849-54.

17. Auron A, Mhanna MJ. Serum creatinine in very low birth weight infants during their first days of life. J Perinatol. 2006;26(12):755-60.

18. Gallini F, Maggio L, Romagnoli C, Marrocco G, Tortorolo G. Progression of renal function in preterm neonates with gestational age $<$ or $=32$ weeks. Pediatr Nephrol. 2000;15(1-2):119-24.
19. Miall LS, Henderson MJ, Turner AJ, Brownlee KG, Brocklebank JT, Newell SJ, et al. Plasma creatinine rises dramatically in the first 48 hours of life in preterm infants. Pediatrics. 1999;104(6), e76.

20.• Selewski DT, Charlton JR, Jetton JG, Guillet R, Mhanna MJ, Askenazi DJ, et al. Neonatal acute kidney injury. Pediatrics. 2015;136(2):e463-73.

Excellent review on neonatal AKI addressing the challenges associated with development of a consensus definition, key aspects of evaluation and management, outcomes for neonatal $\mathrm{AKI} /$ need for longer followup studies, as well as areas where future research is needed.

21. Brierley J, Carcillo JA, Choong K, Cornell T, Decaen A, Deymann A, et al. Clinical practice parameters for hemodynamic support of pediatric and neonatal septic shock: 2007 update from the American College of Critical Care Medicine. Crit Care Med. 2009;37(2):666-88.

22. Akcan-Arikan A. Pediatric acute kidney injury-deadly now, costly later, and a public health burden. Pediatr Crit Care Med. 2015;16(8):774-5.

23. Davison D, Basu RK, Goldstein SL, Chawla LS. Fluid management in adults and children: core curriculum 2014. Am J Kidney Dis. 2014;63(4):700-12.

24. Yunos NM, Bellomo R, Hegarty C, Story D, Ho L, Bailey $\mathrm{M}$. Association between a chloride-liberal vs chloriderestrictive intravenous fluid administration strategy and kidney injury in critically ill adults. JAMA. 2012;308(15):1566-72.

$25 . \bullet \quad$ KDIGO clinical practice guideline for acute kidney injury. Kidney Int.Suppl. 2012;2(1):1-138.

KDIGO Clinical Practice Guideline for the prevention and nondialytic treatment of AKI including hemodynamic support, pharmacologic therapy, nutritional support, and preventive measures.

26. Friedrich JO, Adhikari N, Herridge MS, Beyene J. Metaanalysis: low-dose dopamine increases urine output but does not prevent renal dysfunction or death. Ann Intern Med. 2005;142(7):510-24.

27.• Goldstein SL. Fluid management in acute kidney injury. J Intensive Care Med. 2014;29(4):183-9.

Excellent article addressing the goal directed phases of fluid management during AKI.

28. Bouchard J, Soroko SB, Chertow GM, Himmelfarb J, Ikizler TA, Paganini EP, et al. Fluid accumulation, survival and recovery of kidney function in critically ill patients with acute kidney injury. Kidney Int. 2009;76(4):422-7.

29. Arikan AA, Zappitelli M, Goldstein SL, Naipaul A, Jefferson LS, Loftis LL. Fluid overload is associated with impaired oxygenation and morbidity in critically ill children. Pediatr Crit Care Med. 2012;13(3):253-8.

30. Goldstein SL, Somers MJ, Baum MA, Symons JM, Brophy PD, Blowey D, et al. Pediatric patients with multi-organ dysfunction syndrome receiving continuous renal replacement therapy. Kidney Int. 2005;67(2):653-8.

31. Foland JA, Fortenberry JD, Warshaw BL, Pettignano R, Merritt RK, Heard ML, et al. Fluid overload before 
continuous hemofiltration and survival in critically ill children: a retrospective analysis. Crit Care Med. 2004;32(8):1771-6.

32. Kaur S, Jain S, Saha A, Chawla D, Parmar VR, Basu S, et al. Evaluation of glomerular and tubular renal function in neonates with birth asphyxia. Ann Trop Paediatr. 2011;31(2):129-34.

33. Karlowicz MG, Adelman RD. Nonoliguric and oliguric acute renal failure in asphyxiated term neonates.

Pediatr Nephrol. 1995;9(6):718-22.

34. Hankins GD, Koen S, Gei AF, Lopez SM, Van Hook JW, Anderson GD. Neonatal organ system injury in acute birth asphyxia sufficient to result in neonatal encephalopathy. Obstet Gynecol. 2002;99(5 Pt 1):688-91.

35. Bakr AF. Prophylactic theophylline to prevent renal dysfunction in newborns exposed to perinatal asphyxia-a study in a developing country. Pediatr Nephrol. 2005;20(9):1249-52.

36. Eslami Z, Shajari A, Kheirandish M, Heidary A. Theophylline for prevention of kidney dysfunction in neonates with severe asphyxia. Iran J Kidney Dis. 2009;3(4):222-6.

37. Jenik AG, Ceriani Cernadas JM, Gorenstein A, Ramirez JA, Vain N, Armadans M, et al. A randomized, doubleblind, placebo-controlled trial of the effects of prophylactic theophylline on renal function in term neonates with perinatal asphyxia. Pediatrics. 2000;105(4), E45.

38. Cattarelli D, Spandrio M, Gasparoni A, Bottino R, Offer C, Chirico G. A randomised, double blind, placebo controlled trial of the effect of theophylline in prevention of vasomotor nephropathy in very preterm neonates with respiratory distress syndrome. Arch Dis Child Fetal Neonatal Ed. 2006;91(2):F80-4.

39. Al-Wassia H, Alshaikh B, Sauve R. Prophylactic theophylline for the prevention of severe renal dysfunction in term and post-term neonates with perinatal asphyxia: a systematic review and meta-analysis of randomized controlled trials. J Perinatol.

2013;33(4):271-7.

Review article and meta-analysis of 4 randomized controlled trials demonstrating that use of prophylactic theophylline soon after birth is associated with a lower incidence of severe AKI.

40. Susantitaphong P, Alfayez M, Cohen-Bucay A, Balk EM, Jaber BL. Therapeutic hypothermia and prevention of acute kidney injury: a meta-analysis of randomized controlled trials. Resuscitation. 2012;83(2):159-67.

41. Rhone ET, Carmody JB, Swanson JR, Charlton JR. Nephrotoxic medication exposure in very low birth weight infants. J Matern Fetal Neonatal Med. 2014;27(14):1485-90.

Single center study of very low birth weight infants which quantifies the rate of nephrotoxin exposure.Infants in this study had a high rate of exposure to nephrotoxins.

42. Perez KM, Laughon MM. What is new for patent ductus arteriosus management in premature infants in 2015? Curr Opin Pediatr. 2015;27(2):158-64.

43. Ohlsson A, Walia R, Shah SS. Ibuprofen for the treatment of patent ductus arteriosus in preterm or low birth weight (or both) infants. Cochrane Database Syst Rev. 2015;2, CD003481.

44. Terrin G, Conte F, Oncel MY, Scipione A, McNamara PJ, Simons S, et al. Paracetamol for the treatment of patent ductus arteriosus in preterm neonates: a systematic review and meta-analysis. Arch Dis Child Fetal Neonatal Ed. 2015;17.

45. Eyler RF, Mueller BA, Medscape. Antibiotic dosing in critically ill patients with acute kidney injury. Nat Rev Nephrol. 2011;7(4):226-35.

46. Bagshaw SM, Bellomo R, Kellum JA. Oliguria, volume overload, and loop diuretics. Crit Care Med. 2008;36(4 Suppl):S172-8.

47. Mehta RL, Pascual MT, Soroko S, Chertow GM, PICARD Study Group. Diuretics, mortality, and nonrecovery of renal function in acute renal failure. JAMA. 2002;288(20):2547-53.

48. Oliveros M, Pham JT, John E, Resheidat A, Bhat R. The use of bumetanide for oliguric acute renal failure in preterm infants. Pediatr Crit Care Med.

2011;12(2):210-4.

49. Bunchman TE. Acute kidney injury treatment and the optimization of diuretics in newborns. Pediatr Crit Care Med. 2011;12(2):234-5.

50. Merheb R, Kruzer K, Mhanna M. The effect of bumetanide in extremely low birth weight infants with acute kidney injury during their first weeks of life. J Clin Pediatr Nephrol. 2014;2(1):53-63.

51. Alparslan C, Yavascan O, Bal A, Kanik A, Kose E, Demir $\mathrm{BK}$, et al. The performance of acute peritoneal dialysis treatment in neonatal period. Ren Fail. 2012;34(8):1015-20.

52. Stojanovic V, Bukarica S, Doronjski A, Marinkovic S. Peritoneal dialysis in neonates with extremely low body weight at birth: new modality of using IV cannula for peritoneal access. Iran J Pediatr. 2013;23(6):71820.

53. Oyachi N, Obana K, Kimura S, Kubo M, Naito A, Nemoto A. Use of a flexible Blake(R) silicone drains for peritoneal dialysis in the neonatal intensive care unit. Pediatr Int. 2011;53(3):417-8.

54. Yu JE, Park MS, Pai KS. Acute peritoneal dialysis in very low birth weight neonates using a vascular catheter. Pediatr Nephrol. 2010;25(2):367-71.

55. Harshman LA, Muff-Luett M, Neuberger ML, Dagle JM, Shilyansky J, Nester CM, et al. Peritoneal dialysis in an extremely low-birth-weight infant with acute kidney injury. Clin Kidney J. 2014;7(6):582-5.

56. Raaijmakers R, Schroder CH, Gajjar P, Argent A, Nourse $P$. Continuous flow peritoneal dialysis: first experience in children with acute renal failure. Clin J Am Soc Nephrol. 2011;6(2):311-8.

57. Sutherland SM, Alexander SR. Continuous renal replacement therapy in children. Pediatr Nephrol. 2012;27(11):2007-16.

Excellent review of the technical aspects of providing CRRT to children including issues related to vascular access, choice of membrane filter, dosing of CRRT, and anticoagulation. 
58. Hackbarth R, Bunchman TE, Chua AN, Somers MJ, Baum M, Symons JM, et al. The effect of vascular access location and size on circuit survival in pediatric continuous renal replacement therapy: a report from the PPCRRT registry. Int J Artif Organs. 2007;30(12):111621.

59. El Masri K, Jackson K, Borasino S, Law M, Askenazi D, Alten J. Successful continuous renal replacement therapy using two single-lumen catheters in neonates and infants with cardiac disease. Pediatr Nephrol. 2013;28(12):2383-7.

60. Brophy PD, Mottes TA, Kudelka TL, McBryde KD, Gardner JJ, Maxvold NJ, et al. AN-69 membrane reactions are $\mathrm{pH}$-dependent and preventable. Am J Kidney Dis. 2001;38(1):173-8.

61.• Askenazi D, Ingram D, White S, Cramer M, Borasino S, Coghill C, et al. Smaller circuits for smaller patients: improving renal support therapy with Aquadex ${ }^{\mathrm{TM}}$. Pediatric nephrology (Berlin, Germany) (in press).

This article reports the experience from a 12 patient case series in which an ultrafiltration machine was used to provide CVVH to small infants.

62.• Coulthard MG, Crosier J, Griffiths C, Smith J, Drinnan $M$, Whitaker $\mathrm{M}$, et al. Haemodialysing babies weighing $<8 \mathrm{~kg}$ with the Newcastle Infant Dialysis and Ultrafiltration System (NIDUS): comparison with peritoneal and conventional haemodialysis. Pediatr Nephrol. 2014;29(10):1873-81.

This article describes the NIDUS system, uniquely designed with a syringe pump mechanism and small extracorporeal volume $(6.5 \mathrm{ml})$ to provide HD or CVVHD to neonates as small as $0.8 \mathrm{~kg}$ using a single lumen catheter without the need for a blood prime. The article discusses the CVVHD system, the solute clearance which can be achieved, as well as the precise fluid balance achieved in a case series of patients.

63. Ronco C, Garzotto F, Ricci Z. CA.R.PE.DI.E.M. (CardioRenal Pediatric Dialysis Emergency Machine): evolution of continuous renal replacement therapies in infants. A personal journey. Pediatr Nephrol. 2012;27(8):1203-11.

64.• Ronco C, Ricci Z. Pediatric continuous renal replacement: 20 years later. Intensive Care Med. 2015;41(6):985-93.

Excellent article summarizing the history and evolution of pediatric CRRT and how this led to the development of machinery for neonatal CRRT.

65. Ronco C, Ricci Z, Goldstein SL. (R)evolution in the management of acute kidney injury in newborns. Am J Kidney Dis. 2015;66(2):206-11.

66. Zwiers AJ, De Wildt SN, Hop WC, Dorresteijn EM, Gischler SJ, Tibboel D, et al. Acute kidney injury is a frequent complication in critically ill neonates receiving extracorporeal membrane oxygenation: a 14-year cohort study. Crit Care. 2013;17(4):R151.

Large single center cohort study of the incidence and clinical course of AKI amongst full term neonates receiving ECMO, but not hemofiltration, between 1992-2006.

67. Smith AH, Hardison DC, Worden CR, Fleming GM, Taylor MB. Acute renal failure during extracorporeal support in the pediatric cardiac patient. ASAIO J. 2009;55(4):412-6.

68. Blijdorp K, Cransberg K, Wildschut ED, Gischler SJ, Jan Houmes R, Wolff ED, et al. Haemofiltration in newborns treated with extracorporeal membrane oxygenation: a case-comparison study. Crit Care. 2009;13(2):R48.

69. Selewski DT, Cornell TT, Lombel RM, Blatt NB, Han YY, Mottes T, et al. Weight-based determination of fluid overload status and mortality in pediatric intensive care unit patients requiring continuous renal replacement therapy. Intensive Care Med. 2011;37(7):1166-73.

70. Swaniker F, Kolla S, Moler F, Custer J, Grams R, Barlett $\mathrm{R}$, et al. Extracorporeal life support outcome for 128 pediatric patients with respiratory failure. J Pediatr Surg. 2000;35(2):197-202.

71. Anderson III HL, Coran AG, Drongowski RA, Ha HJ, Bartlett RH. Extracellular fluid and total body water changes in neonates undergoing extracorporeal membrane oxygenation. J Pediatr Surg. 1992;27(8):1003-7. discussion 1007-8.

72. Kelly Jr RE, Phillips JD, Foglia RP, Bjerke HS, Barcliff LT, Petrus L, et al. Pulmonary edema and fluid mobilization as determinants of the duration of ECMO support. J Pediatr Surg. 1991;26(9):1016-22.

73. Selewski DT, Cornell TT, Blatt NB, Han YY, Mottes T, Kommareddi M, et al. Fluid overload and fluid removal in pediatric patients on extracorporeal membrane oxygenation requiring continuous renal replacement therapy. Crit Care Med. 2012;40(9):2694-9.

Retrospective single center study demonstrating an association between higher degree of fluid overload at initiation of CRRT and increased mortality. The study also suggests that correction of signficant fluid overload, once established, may not be as beneficial as the early initiation of CRRT prior to the development of significant fluid overload.

74.• Askenazi DJ, Selewski DT, Paden ML, Cooper DS, Bridges BC, Zappitelli M, et al. Renal replacement therapy in critically ill patients receiving extracorporeal membrane oxygenation. Clin J Am Soc Nephrol. 2012;7(8):1328-36.

Excellent, comprehensive review of AKI as a complication of ECMO discussing the epidemiology and pathophysiology of AKI in ECMO patients, indications for RRT when on ECMO, and highlighting technical considerations when performing CRRT for ECMO patients.

75. Sasser WC, Robert SM, Askenazi DJ, O'Meara LC, Borasino S, Alten JA. Peritoneal dialysis: an alternative modality of fluid removal in neonates requiring extracorporeal membrane oxygenation after cardiac surgery. J Extra Corpor Technol. 2014;46(2):157-61.

76. Zwiers AJ, Cransberg K, van Rosmalen J, Wildschut ED, Tibboel D, de Wildt SN. Loop diuretics are an independent risk factor for acute kidney injury in children on extracorporeal membrane oxygenation with preemptive continuous hemofiltration. Intensive Care Med. 2014;40(4):627-8. 